\title{
Intermittent Switching between Soliton Dynamic States in a Perturbed Sine-Gordon Model
}

Sørensen, Mads Peter; Arley, N.; Christiansen, Peter Leth; Parmentier, R. D.; Skovgaard, Ove

Published in:

Physical Review Letters

Link to article, DOI:

10.1103/PhysRevLett.51.1919

Publication date:

1983

Document Version

Publisher's PDF, also known as Version of record

Link back to DTU Orbit

Citation (APA):

Sørensen, M. P., Arley, N., Christiansen, P. L., Parmentier, R. D., \& Skovgaard, O. (1983). Intermittent Switching between Soliton Dynamic States in a Perturbed Sine-Gordon Model. Physical Review Letters, 51(20), 19191922. https://doi.org/10.1103/PhysRevLett.51.1919

\section{General rights}

Copyright and moral rights for the publications made accessible in the public portal are retained by the authors and/or other copyright owners and it is a condition of accessing publications that users recognise and abide by the legal requirements associated with these rights.

- Users may download and print one copy of any publication from the public portal for the purpose of private study or research.

- You may not further distribute the material or use it for any profit-making activity or commercial gain

- You may freely distribute the URL identifying the publication in the public portal 


\title{
Intermittent Switching between Soliton Dynamic States in a Perturbed Sine-Gordon Model
}

\author{
M. P. Soerensen, N. Arley, P. L. Christiansen, R. D. Parmentier, (a) and O. Skovgaard \\ Laboratory of Applied Mathematical Physics, The Technical University of Denmark, DK-2800 Lyngby, Denmark
} (Received 6 June 1983)

\begin{abstract}
Chaotic intermittency between soliton dynamic states has been found in a perturbed sine-Gordon system in the absence of an external ac driving term. The system is a model of a long Josephson oscillator with constant loss and bias current in an external magnetic field. The results predict the existence of a current step between the first two Fiske steps in the current-voltage characteristic. A simple probability model demonstrates an asymmetry in the statistical nature of the switching in the two directions.
\end{abstract}

PACS numbers: $74.50 .+r, 05.40 .+j, 84.30 . \mathrm{Ng}, 85.25 .+\mathrm{k}$

Chaos phenomena have been found for the rfand dc-current driven small Josephson junction described by the resistively shunted junction (RSJ) model. ${ }^{1}$ Recently Ben-Jacob et al. and Yeh and Kao reported on intermittent chaos in the numerical solution of this model..$^{2}$ For a long Josephson junction, the perturbed sine-Gordon equation (SGE) with spatially uniform or nonuniform oscillating driving forces and linear damping also gives rise to chaos in time and spacetime. ${ }^{3}$ Detailed numerical investigations have revealed subharmonic generation caused by soliton motion in a long Josephson junction in a constant external magnetic field modeled by the perturbed SGE without an external ac driving term in the current bias. ${ }^{4}$ In the present Letter we demonstrate a new chaotic intermittency phenomenon between two dynamic states of this model. The two states correspond physically to the first two Fiske steps (FS1 and FS2, respectively) in the current-voltage characteristic of the junction.

The mathematical model studied is ${ }^{5}$

$$
\begin{aligned}
& \varphi_{x x}-\varphi_{t t}-\sin \varphi=\alpha \varphi_{t}-\gamma, \\
& \varphi_{x}(0, t)=\varphi_{x}(L, t)=\eta .
\end{aligned}
$$

Here $\varphi$ is the usual Josephson phase variable, $x$ is distance normalized to the Josephson penetration depth $\lambda_{J}$, and $t$ is time normalized to the inverse of the Josephson plasma frequency $\omega_{0}$. The $\gamma$ term represents a uniformly distributed constant bias current normalized to the maximum zero-voltage (Josephson) current. The term in $\alpha$ represents quasiparticle loss. The constant $\eta$ is a normalized measure of the external magnetic field which determines the boundary conditions (1b) at the ends of the junction of normalized length $L$. In this study $L=5, \alpha=0.252, \eta=1.25$, and $\gamma$ is varied in the range $\gamma=0.45-0.55$. Equations (1) were integrated from appropriate initial conditions [similar to Eqs. (2) of Ref. 4 or con- tinuations from runs done at nearby points in parameter space] for long periods of time (typically $t \sim 10000$ ) by means of the implicit finitedifference method described in detail in Ref. 5, with space and time intervals set equal to 0.05 and 0.025 , respectively. Numerical accuracy and stability were verified by halving the space and time intervals.

In a narrow range of relatively low $\gamma$ values $(\gamma$ $=0.450-0.454)$ the solution develops stably into the FS1 solution illustrated in Fig. 1(a). In physical terms FS1 corresponds to a situation in which a soliton propagates in the field-aided direction, and is reflected at $x=0$ as a localized plasma wave because of the energy loss at this boundary. This plasma wave then moves in the opposite direction and is reflected as a soliton at $x=L$ as a result of the energy injection here by the magnetic field. [An unambiguous interpretation of Fig. 1(a) in the schematic terms shown in Fig. 1(c) may be established by examining together $\varphi(x, t)$, $\varphi_{x}(x, t)$, and $\varphi_{t}(x, t)$.] The soliton then resumes propagation as before the reflections. ${ }^{4,6,7}$ On the average this cycle lasts the time $\bar{t}_{\mathrm{F}} \cong 12$ (at $\gamma$ $=0.454$ ). For $\gamma<0.450$ the junction switches into a static zero-voltage state where $\varphi_{t}=0$.

For relatively high $\gamma$ values $(\gamma=0.500-0.540)$ the solution develops stably into the F S2 solution corresponding to a situation in which a soliton and a localized plasma wave travel in opposite directions at the same time [ shown numerically in Fig. 1(b) and schematically in Fig. 1(c)]. The average length of this cycle was estimated to be $\bar{t}_{\mathrm{FS} 2} \cong 6$ (at $\left.\gamma=0.500\right)$. For $\gamma>0.540$ the junction switches to FS3.

For intermediate $\gamma$ values $(\gamma=0.456-0.490)$ the junction exhibits chaotic intermittency between FS1 and FS2. The intermittency is shown in Fig. 2 for $\gamma=0.480$, where it is evidenced by changes in the average slope of $\varphi$ versus $t$ (on the FS1 portions $\left\langle\varphi_{t}\right\rangle \cong 0.6$ and on the FS2 por- 

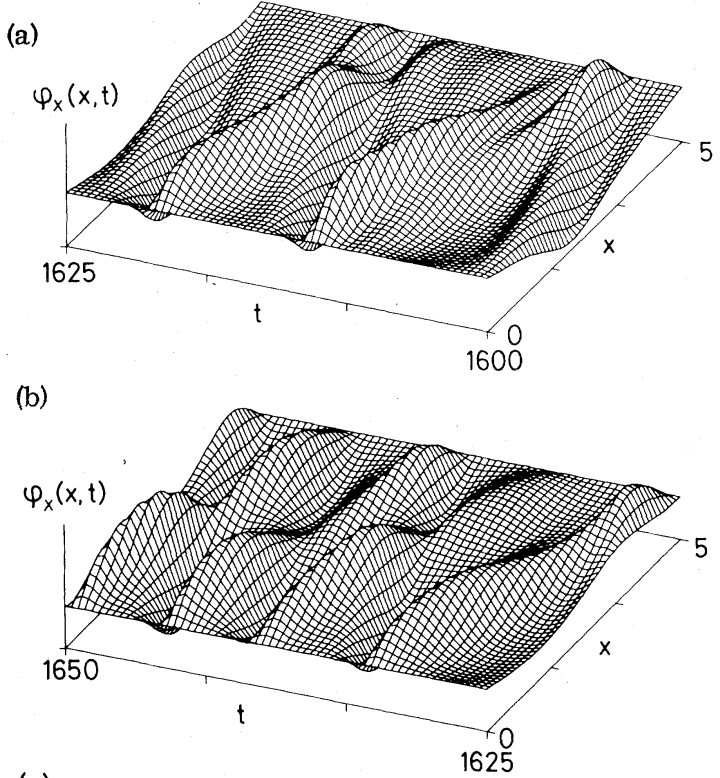

(c)

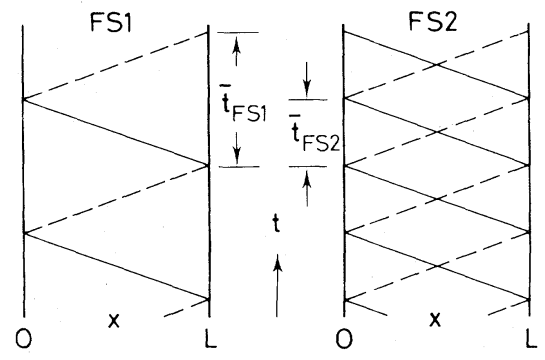

FIG. 1. $\varphi_{x}(x, t)$ on (a) FS1 and (b) FS2 portions of solution in intermittency region $(\gamma=0.480)$. (c) Schematic representations of (a) and (b) showing soliton (solid lines) and plasma-wave (dashed lines) trajectories. A switch from FS1 to FS2 is seen at $t \cong 1625$.

tions $\left.\left\langle\varphi_{t}\right\rangle \cong 1.0\right)$. The switch from FS2 to FS1 occurs when the plasma oscillation fails to generate a soliton at $x=L$. Conversely, a switch from FS1 to FS2 may occur when an extra soliton is generated at $x=L$ during the FS1 cycle. The power spectrum (inset in Fig. 2) of $\varphi_{t}(0, t)$, normalized to $\varphi_{t}^{2}=4 \times 10^{-7}$, was obtained by a fast Fourier transform over the time interval 16003238 by use of a Hamming window. ${ }^{8}$ Since loading effects are not included in the model, the power levels in this spectrum represent ideal, available values. Translation into physically measurable quantities requires a knowledge of the junctionto-microwave circuit coupling (to set the physical power scale) and the junction plasma frequency (to set the physical frequency scale). The spectrum shows numerous frequency components among which the two dominant lines at $t^{-1}=0.10$ and 0.17 may be ascribed to the soliton motion

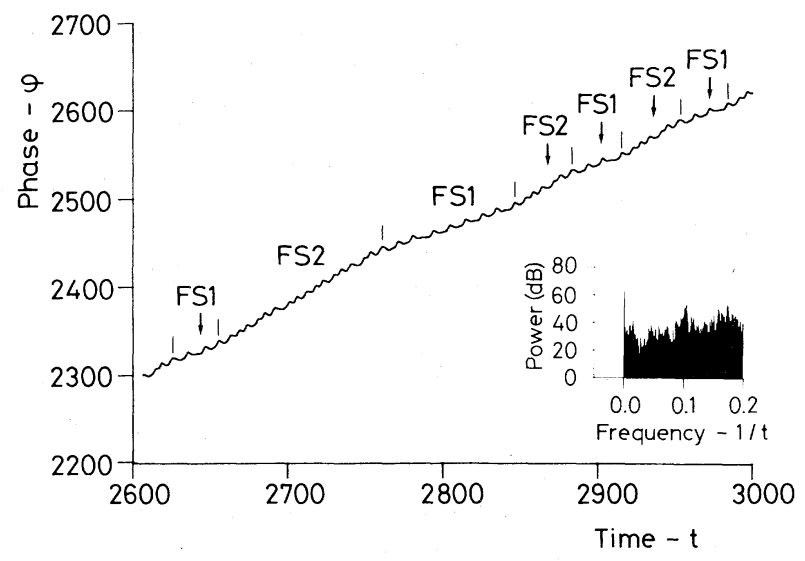

FIG. 2. Phase vs time at the left end $(x=0)$ showing intermittency between FS1 and FS2 at $\gamma=0.480$. Inset: Power spectrum of $\varphi_{t}(0, t)$, normalized to $\varphi_{t}{ }^{2}=4$ $\times 10^{-7}$, for $t=1600-3238$, with frequency measured in units of the Josephson plasma frequency.

on FS1 and FS2, respectively. The average times the junction remains on FS1 and FS2 were found to be $\bar{T}_{\mathrm{FS} 1} \cong 46$ and $\bar{T}_{\mathrm{ES} 2} \cong 52$ (at $\left.\gamma=0.480\right)$. We have found that $\overline{\boldsymbol{T}}_{\mathrm{F} \mathrm{S}_{1}}$ and $\bar{T}_{\mathrm{FS} 2}$ remain stationary in time at these values. The corresponding lines are barely visible in the low end of the power spectrum.

For a particularly long time interval shown in Fig. $3(t=1902-2079)$ the junction remained on FS2. Around $t=1960$ the development of aperiodicity is observed. The insets (a) and (b) show the power spectrum for the time intervals 19021951 and 1984-2079, respectively, again ob-

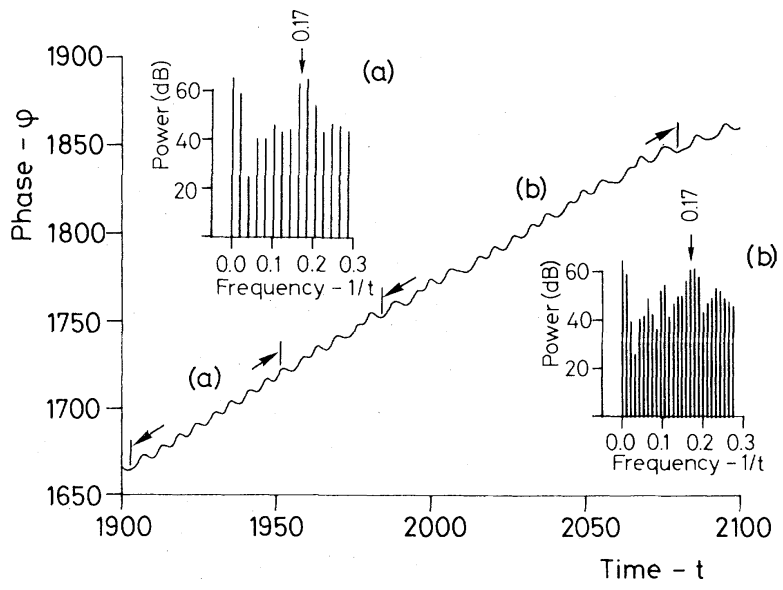

FIG. 3. Phase vs time at the left end $(x=0)$ for a particularly long operation on FS2 at $\gamma=0.480$. Insets: Power spectra of $\varphi_{t}(0, t)$, normalized to $\varphi_{t}{ }^{2}=4 \times 10^{-7}$, for (a) $t=1902-1951$ and (b) $t=1984-2070$. The fundamental frequency is $f_{0}=0.17$. 


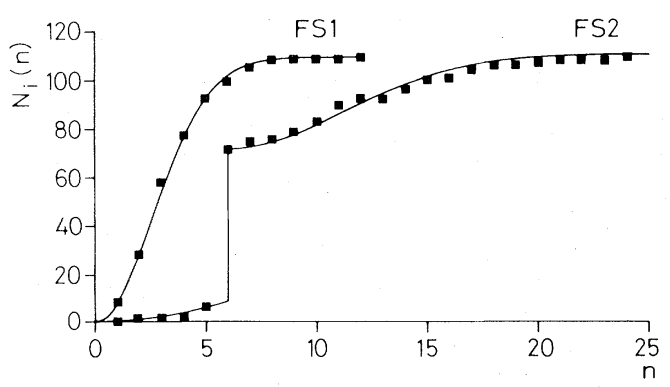

FIG. 4. Abscissa: Length of time interval on FS1 and FS2 measured in terms of number of cycles, $n$, of lengths $\bar{t}_{\mathrm{FS} 1}$ and $\bar{t}_{\mathrm{FS} 2}$, respectively. Ordinate: Dots show number of intervals, $N_{i}(n)$, with $i=1,2$, shorter than or equal to $n$. Full curves result from theoretical estimates with $p_{12}(t)=\left(1.0 \times 10^{-3}\right) t, p_{21}(t)=\left(1.2 \times 10^{-4}\right) t$ for $n<6, p_{21}(t)=\left(0.95 \times 10^{-5}\right) t$ for $n>6$. Bias $\gamma=0.480$.

tained by use of a Hamming window. In inset (a) we observe the dominant frequency at $t^{-1} \cong 0.17$ and a relatively low content of subharmonics. In inset (b) subharmonics have developed at approximately $\frac{1}{3}$ and $\frac{2}{3}$ of this frequency. A similar building up of subharmonics is not seen while the junction operates on FS1.

Figure 4 (dots) shows the accumulated distributions, $N_{1}(n)$ and $N_{2}(n)$, of the lengths of the time intervals the junction operates on FS1 and FS2 for 217 switches between the two steps during a run of over 10000 time units. Here $n$ is $t / \bar{t}_{\mathrm{FS} 1}$ and $t / \bar{t}_{\mathrm{FS} 2}$, respectively. To analyze this situation, let the probability that the junction switches from FS1 to FS2 in the time interval $[t, t+d t]$, where $t$ is the time after the last switch, be $p_{12}(t) d t$. Furthermore, if all the switches are independent, then the switching is a Poisson process. Consequently, the probability $P_{1}(t)$ that the oscillator switches from FS1 to FS2 within the time $t$ becomes $P_{1}(t)=1-\exp \left[-\int_{0}{ }^{t} p_{12}(\tau) d \tau\right]$. As suming $p_{12}(t)=p t$, with $p=1.0 \times 10^{-3}$, we get for FS1 the fit $N_{1}=P_{1}\left(n \bar{t}_{\mathrm{FS} 1}\right) N_{1 \max }$, where $N_{1 \max }$ is the total number of intervals on FS1, shown in Fig. 4 (full curve). The agreement between the numerical data and this simple, but arbitrary, probability model is quite good.

For FS2 a jump of 63 in $N_{2}(n)$ at $n=6$ is observed in our 10000-time-unit run. Only by use of a transition probability, $p_{21}(t)$, containing 0.96 times a unit impulse at $n=6$, besides a linear term, do we obtain the fit shown in Fig. 4. The difference between the two results clearly demonstrates an asymmetry in the statistical nature of the switching in the two directions. The reason for this fact is not known. A possible cause

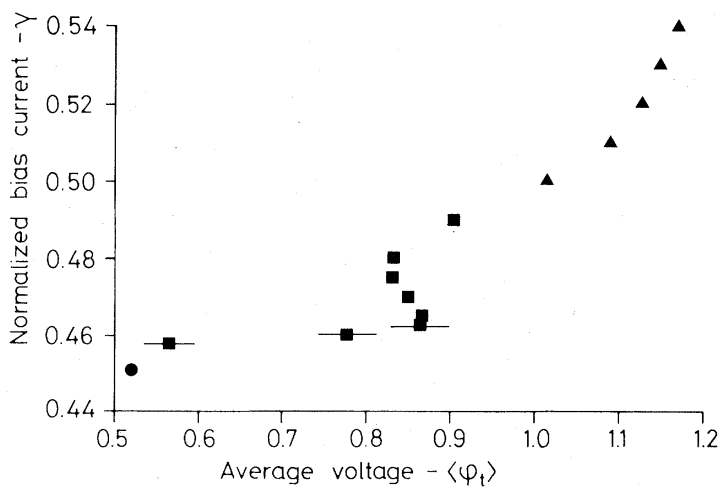

FIG. 5. Detail of current-voltage characteristic for $L=5, \alpha=0.252, \eta=1.25$. FS1 (circles), FS1 $\frac{1}{2}$ (squares), and FS2 (triangles). Error limits for $\left\langle\varphi_{t}\right\rangle$ are indicated by horizontal bars when larger than 0.01 .

may be an interference between the subharmonics $\frac{1}{3}$ and $\frac{2}{3}$ which build up on FS2, as seen in Fig. 3 , but the question certainly requires further study.

Finally, Fig. 5 shows the resulting currentvoltage characteristic for the $\gamma$ interval covering FS1, the intermittency region, and FS2. At the average voltage $\left\langle\varphi_{t}\right\rangle \cong 0.83$ a jump in the current (from $\gamma=0.462$ to $\gamma=0.480$ ) occurs. We propose the name "FS1 $\frac{1}{2}$ " for this portion of the characteristic. We have checked that the values of $\left\langle\varphi_{t}\right\rangle$ are stationary in time. Thus it should be possible to detect FS1 $\frac{1}{2}$ experimentally. Recent measurements by Cirillo, Costabile, and Parmentier ${ }^{9}$ have in fact perhaps revealed such structures.

We thank A. C. Scott and N. F. Pedersen for stimulating discussions. The financial support of the Danish Natural Science Research Council, the Thomas B. Thriges Fond, and the European Research Office of the U. S. Army through Contract No. DAJA $37-82-\mathrm{C}-0057$ is acknowledged.

\footnotetext{
(a) Permanent address: Istituto di Fisica, Università di Salerno, I-84100 Salerno, Italy.

${ }^{1}$ B. A. Huberman, J. P. Crutchfield, and N. H. Packard, Appl. Phys. Lett. 37, 750 (1980); N. F. Pedersen and A. Davidson, Appl. Phys. Lett. 39, 830 (1981); M. Cirillo and N. F. Pedersen, Phys. Lett. 90A, 150 (1982); R. L. Kautz, IEEE Trans. Magn. 19, 465 (1983); A. H. MacDonald and M. Plischke, Phys. Rev. B 27, 201 (1983).

${ }^{2}$ E. Ben-Jacob, I. Goldhirsch, Y. Imry, and S. Fishman, Phys. Rev. Lett. $\underline{49}, 1599$ (1982); W. J. Yeh and
} 
Y. H. Kao, Appl. Phys. Lett. 42, 299 (1983).

${ }^{3}$ J. C. Eilbeck, P. S. Lomdahl, and A. C. Newell, Phys. Lett. 87A, 1 (1981); D. Bennett, A. R. Bishop, and S. E. Trullinger, Z. Phys. B74, 265 (1982); A. R. Bishop, K. Fesser, P. S. Lomdahl, W. C. Kerr, M. B. Williams, and S. E. Trullinger, Phys. Rev. Lett. $\underline{50}$, 1095 (1983); M. Imada, J. Phys. Soc. Jpn. $\underline{52}, 1946$ (1983).

${ }^{4}$ M. P. Soerensen, P. L. Christiansen, R. D. Parmentier, and O. Skovgaard, Appl. Phys. Lett. $\underline{42}, 739$ (1983).
${ }^{5}$ P. S. Lomdahl, O. H. Soerensen, and P. L. Christiansen, Phys. Rev. B 25, 5737 (1982).

${ }^{6}$ B. Dueholm, E. Joergensen, O. A. Levring, J. Mygind, N. F. Pedersen, M. R. Samuelsen, O. H. Olsen, and M. Cirillo, Physica (Utrecht) 108B, 1303 (1981).

${ }^{7}$ S. N. Erné, A. Ferrigno, and R. D. Parmentier, Phys. Rev. B 27, 5440 (1983).

${ }^{8}$ R. W. Hamming, Digital Filters (Prentice-Hall, Englewood Cliffs, N.J., 1983), 2nd ed.

${ }^{9}$ M. Cirillo, G. Costabile, and R. D. Parmentier, unpublished. 